DRAFT VERSION JULY 7, 2021

Preprint typeset using LATEX style emulateapj v. 11/12/01

\title{
FIRST DETECTION OF MILLIMETER DUST EMISSION FROM BROWN DWARF DISKS
}

\author{
R. KLEIN \\ Astrophysical Institute and University Observatory (AIU) Jena, Schillergäßchen 2-3, D-07745 Jena, Germany \\ rklein@astro.uni-jena.de \\ D. Apai, I. Pascucci, Th. Henning \\ Max Planck Institute for Astronomy, Königstuhl 17, D-69117 Heidelberg, Germany \\ apai@mpia-hd.mpg.de;pascucci@mpia-hd.mpg.de;henning@mpia-hd.mpg.de \\ AND \\ L. B. F. M. WATERS \\ Astronomical Institute "Anton Pannekoek", University of Amsterdam, Kruislaan 403, NL-1098 SJ Amsterdam, Netherlands \\ Institute of Astronomy, Katholieke Universiteit Leuven, Celestijnenlaan 200B, B3001 Heverlee, Belgie \\ rensw@science.uva.nl \\ Draft version July 7, 2021
}

\begin{abstract}
We report results from the first deep millimeter continuum survey targeting Brown Dwarfs (BDs). The survey led to the first detection of cold dust in the disks around two young BDs (CFHT-BD-Tau 4 and IC 348 613), with deep JCMT and IRAM observations reaching flux levels of a few mJy. The dust masses are estimated to be a few Earth masses assuming the same dust opacities as usually applied to T Tauri stars.
\end{abstract}

Subject headings: accretion, accretion disks - circumstellar matter — stars: formation - stars: low-mass, brown dwarfs - stars: individual (CFHT-BD-Tau 4, IC 348 613)

\section{INTRODUCTION}

Several near-infrared surveys (Muench et al. 2001; Oliveira et al. 2002; Liu et al. 2003; Jayawardhana et al. 2003) and midinfrared measurements (Comerón et al. 2000; Persi et al. 2000; Testi et al. 2002; Natta et al. 2002; Apai et al. 2002) indicate the presence of disks around Brown Dwarfs (BDs). In contrast to infrared emission, submillimeter and millimeter emission is certainly always optically thin and is an excellent measure for the total dust mass.

An early attempt to detect millimetre continuum emission also from very low-mass stars and suspected BDs was done by Andre \& Montmerle (1994). Probably the most sensitive observation of this kind was carried out by Carpenter (2002) using the OVRO interferometer, however at a relatively long wavelength. We carried out the first successful search for dust continuum emission associated with confirmed BDs, using the bolometer arrays SCUBA at the JCMT and MAMBO at the IRAM 30-m telescope.

The survey led to the detection of circumstellar dust around the two young BDs CFHT-BD-Tau 4 and IC 348 613, which have ages below 10 Myrs. In the case of field BDs we obtained upper mass limits of a few Moon masses of dust. For BDs in the Pleiades the mass limits are less strict and range 4 and 7 Earth masses. We should note that the data presented for CFHT-BDTau 4 together with other ground-based and ISO data allowed the first detailed discussion of a complete spectral energy distribution of a BD, ranging from optical to millimeter wavelengths (Pascucci et al. 2003).

The detection of these amounts of circumstellar material around two young BDs makes the formation of planets or even planetary systems around BDs a possibility. Therefore, search strategies for planets should include BDs. In the case of imaging surveys, they might even be the best targets.

In addition, the detection of significant amount of circum- stellar dust carries important information about the formation processes of BDs. These detections, together with the discovery of quite a number of BD binaries (Bouy et al. 2003; Burgasser et al. 2003), do not support fragmentation of circumstellar disks as the general process for BD formation. Other formation scenarios for BDs (Bate et al. 2003; Reipurth \& Clarke 2001; Watkins et al. 1998) include ejection from multiple systems and erosion of star-forming cloudlets by stellar winds and UV radiation from massive stars. Disks will certainly have different structures depending on the formation mechanism. However, statistics is still poor and information about the disk structure from interferometric observations are needed before one can put more definite observational constraints on BD formation scenarios.

\section{OBSERVATIONS}

To search for circumstellar material around BDs, we selected relatively young BDs with an age of a few Myrs because these objects should have the highest probability to be associated with disk material. The nine selected objects are located in Taurus, the $\sigma$ Orionis cluster, IC 348, and the Upper Scorpius OB association (Martín et al. 2001; Béjar et al. 2001; Najita et al. 2000; Luhman 1999; Ardila et al. 2000). For the first three regions, additional selection criteria were the previous detection of $\mathrm{H} \alpha$ emission (Martín et al. 2001), the presence of X-ray emission (Mokler \& Stelzer 2002; Preibisch \& Zinnecker 2001), and the requirement that the objects should be as isolated as possible in order to avoid confusion during the observations with singledish telescopes.

The three BDs in the Taurus star-forming region are among the youngest BDs of our target list. They have ages of about 1 Myr (Martín et al. 2001). The object CFHT-BD-Tau 4 shows the highest $\mathrm{H} \alpha$ emission among the Taurus BDs (Martín et al. 2001), emits X-ray radiation (Mokler \& Stelzer 2002) and shows mid-infrared excess emission (Pascucci et al. 2003). 
The second group, we selected, are objects in the Pleiades which have a distance of $116 \mathrm{pc}$ and an age of $0.12 \mathrm{Gyr}$. The objects are considerably older than the first group of BDs. In addition, we searched for dust emission from very nearby field BDs. On the average, these objects are older than the first group, too, but the actual age determination contains large uncertainties. ble 1 .

The targets with their ages and distances are compiled in Ta-

The observations were carried out at the James Clerk Maxwell Telescope (JCMT) on Mauna Kea, Hawai'i, and at the IRAM $30 \mathrm{~m}$ telescope, Pico Veleta, Spain. At the JCMT we used the 37-channel array camera SCUBA (Holland et al. 1999) at a wavelength of $850 \mu \mathrm{m}$. At the IRAM 30-m telescope, we performed the observations at $1.3 \mathrm{~mm}$ with the 37 -channel array camera MAMBO (Kreysa et al. 1998). These wavelengths are a good tradeoff between the expected flux and the opacity of the atmosphere.

The observations were performed between March 2002 and January 2003. The chosen observing mode is photometry with the central bolometer of SCUBA and MAMBO, respectively. The background (telluric and astronomical) subtraction is achieved by chopping with the secondary mirror and nodding the telescope. Its level has been estimated from the inner ring consisting of six bolometer pixels.

The measured flux densities and derived upper limits are listed in Table 2. For many sources, we can only derive upper limits since the signal-to-noise-ratio (SNR) is less than three. The upper limits are given as $3 \sigma$ values, where $\sigma$ is the standard deviation of the statistical error.

\section{RESULTS}

We detected millimeter continuum emission associated with two BDs. Both, BD Tau 4 and IC 348 613, were detected at two wavelengths, i.e. with the JCMT at $850 \mu \mathrm{m}$ and with the IRAM $30 \mathrm{~m}$ telescope at $1.3 \mathrm{~mm}$. These detections can be used to estimate the amount of circumstellar matter around these BDs for the first time.

Carpenter (2002) however using the OVRO interferometer to map IC 348 did not detect IC 348613 . The reason is that he mapped at a wavelength of $3 \mathrm{~mm}$. If we extrapolate the measured flux densities to a wavelength of $3 \mathrm{~mm}$, the flux density falls just below the detection limit of the OVRO map.

\subsection{Dust Masses}

The measured flux density $F_{\nu}$ at submillimeter/millimeter wavelengths is certainly optically thin thermal emission by cold dust heated by the BD. The dust mass $M$ can be derived using the formula

$$
M=\frac{F_{\nu} D^{2}}{B_{\nu}(T, \lambda) \kappa_{\nu}(\lambda)},
$$

where $F_{\nu}$ stands for the flux density, $B_{\nu}$ for the Planck function, $T$ for the dust temperature and $\kappa_{\nu}$ for the mass absorption coefficient. The quantity $D$ is the distance to the object. Likewise upper limits for the flux density are translated into upper limits for the amount of circumstellar matter.

Among the above quantities, the distances are well established from trigonometric parallaxes in the case of most of the field BDs. For the young BDs we used the known distances to the clusters/star-forming regions. However, the dust properties $\kappa_{\nu}$ and the dust temperature $T$ need a more thorough discussion.
The targeted BDs have a large spread in age and we would expect different evolutionary stages of the disk material if present at all. Therefore, we will apply two different sets of dust parameters (see, e.g., Henning et al. 1995). The "young" dust parameters will be applied to BDs with ages up to $10 \mathrm{Myr}$. The "debris" dust parameters will be used for the other objects, which are all older than $100 \mathrm{Myr}$. We assume a constant dust temperature as an approximation to the temperature distribution of the circumstellar dust. The next paragraphs discuss the two sets of dust parameters.

"Young" dust: For dust around young BDs, we choose, for the sake of comparison, a mass absorption coefficient of $\kappa_{\nu}=2 \mathrm{~cm}^{2} \mathrm{~g}^{-1}$ at $1.3 \mathrm{~mm}$. The same value of $\kappa_{\nu}$ and a gas-todust ratio of 100 was applied by Beckwith et al. (1990) to derive disk masses for T Tauri disks. For the measurements at $850 \mu \mathrm{m}$, we assume a wavelength dependence of $\kappa_{\nu} \propto \lambda^{-\beta}$ with $\beta=1$, also in accordance with Beckwith et al. (1990). This leads to $\kappa_{\nu}=3 \mathrm{~cm}^{2} \mathrm{~g}^{-1}$ at $850 \mu \mathrm{m}$.

The plausible range of dust temperatures is relatively small. We assume an average temperature of 10 to $20 \mathrm{~K}$ for the dust. This is the range of the mass-averaged dust temperature in the models for the disk around CFHT-BD-Tau 4 discussed by Pascucci et al. (2003).

"Debris" dust: The BDs in the Pleiades and the field objects are presumably older than $100 \mathrm{Myr}$. At this age one can no longer assume a T Tauri-like disk with its dust properties. If there is a disk left it will resemble debris disks like the $\beta$ Pictoris disk. To allow comparisons to submillimeter observations of debris disks, we adopted the mass absorption coefficient used by Dent et al. (2000), i.e. $\kappa_{\nu}=0.4 \ldots 1.7 \mathrm{~cm}^{2} \mathrm{~g}^{-1}$ at $850 \mu \mathrm{m}$. The gas-to-dust mass ratio in debris disks is controversially discussed (Thi et al. 2001; Lecavelier des Etangs et al. 2001). Therefore, we will only give the dust masses in these cases.

Since debris disks are optically thin, the circumstellar dust grains around the old BDs have at least the temperature of interstellar grains, i.e. 20 to $30 \mathrm{~K}$ depending on the composition and the actual interstellar radiation field at the position of the BDs. The heating of the dust by the old BD is negligible, thus, we assume a temperature between 20 to $30 \mathrm{~K}$.

Applying the above discussed dust properties to the millimeter continuum measurements, we obtain the dust masses compiled in Table 2 . The ranges result from the uncertainties in the mass absorption coefficient and the temperature. Furthermore, the millimeter emission has been measured at two wavelengths for the detections. The mass estimates derived from the two measurements differ slightly. For the detections, the masses in Table 2 correspond to the minimum and maximum mass estimates. In the case of non-detections, the mass limits are derived from the $3 \sigma$ flux limits using the dust parameters yielding the highest masses.

The mass of circumstellar dust for CFHT-BD-Tau 4 is between 1.4 and 7.6 $M_{\mathrm{E}}$ (Earth mass) and for IC 348613 the mass ranges between 5.4 and $18 M_{\mathrm{E}}$. The dust is certainly distributed in the form of disks as a detailed analyses of CFHT-BD-Tau 4 by Pascucci et al. (2003) show. The disk masses for the two BDs are $0.4 \ldots 2.4 M_{\mathrm{J}}$ (Jupiter mass) and $1.7 \ldots 5.7 M_{\mathrm{J}}$, if we extrapolate the dust masses to disk masses assuming a gas-to-dust ratio of 100. The upper limits on the circumstellar dust mass around the other young BDs are around a few Earth masses.

The dust mass constraints for the nearby field BDs are even more stringent, reaching upper limits of some Moon masses. Thus, even around the low-luminosity BDs circumstellar disks 
cannot be long-lived. Debris disks around the old BDs, which may be associated with planetary systems, cannot be ruled out, since we were not sensitive down to fractions of Moon masses of dust which is the order of the dust mass around the low-mass main-sequence star $\epsilon$ Eridani (Dent et al. 2000).

\subsection{Disks around young BDs}

One important question about BDs and their disks is the mass ratio of the central objects and the circumstellar material. The BDs' masses themselves (CFHT-BD-Tau 4: $M_{*}=30 \ldots 75 M_{\mathrm{J}}$ 1, Martín et al. 2001; IC 348 613: $M_{*}=20 \ldots 40 M_{\mathrm{J}}$, Preibisch $\&$ Zinnecker 2001) are uncertain as well as the disk masses. Therefore we do not attempt to estimate their ratio here. Still, we note that the observations are consistent with a disk/BD mass ratio of a few percent as it is found for most of the T Tauri stars.

The detection of some Jupiter masses of matter around BDs is especially interesting for extrasolar planet searches. Guenther \& Wuchterl (2003) mention that planets may form around a BD if a disk of enough mass is present. Their radial velocity survey targets very low-mass stars and BDs because these targets are relatively inactive. Furthermore, young planets around BDs would be the easiest to detect by direct imaging because the contrast between the BD and its planet would be much more favorable than for a planet around a higher-mass star.

\section{SUMMARY}

The observing campaign targeting BDs of several populations resulted in detections of millimeter emission associated with two young BDs. The mass estimates for the young BD disks are $0.4 \ldots 2.4 M_{\mathrm{J}}$ and $1.7 \ldots 5.7 M_{\mathrm{J}}$ for CFHT-BD-Tau 4 and IC 348613 , respectively. For the other targets, stringent upper limits on the amount of circumstellar matter were derived from the measured upper limits on the millimeter continuum flux densities. To estimate the dust masses, two sets of dust properties had to be applied: "Young" dust properties to BDs younger than $10 \mathrm{Myr}$ and "debris" dust properties to BDs older than 100 Myr.

The detection of a few Jupiter masses of circumstellar matter around young BDs is an important result. To ensure this mass estimate, the dust properties have to be constrained further. However, a refinement of the dust properties will hardly change the fact that there are substantial amounts of circumstellar material around the two BDs CFHT-BD-Tau 4 and IC 348613 . Thus, the detections make BDs to places of possible planet formation. This fact opens a new set of targets for extrasolar planet searches, especially for direct imaging because of the low contrast between the central object and an prospective planet.

We thank Remo Tilanus for the valuable support before, during and after the JCMT observations. The JCMT is operated by the Joint Astronomy Centre on behalf of the UK Particle Physics and Astronomy Research Council, the Canadian National Research Council and the Netherlands Organization for Scientific Research. The observing run at the JCMT was supported by the Deutsche Forschungs Gemeinschaft (DFG) through grants $\mathrm{Kl} 1330 / 2-1$ and Kl 1330/3-1. DA and IP acknowledge the staff support during the IRAM run 001-02. We thank the referee for the helpful comments on our work. RK acknowledges support through the DFG grant He 1935/15-1.

\section{REFERENCES}

Andre, P. \& Montmerle, T. 1994, ApJ, 420, 837

Apai, D., Pascucci, I., Henning, T., Sterzik, M. F., Klein, R., Semenov, D., Günther, E., \& Stecklum, B. 2002, ApJ, 573, L115

Ardila, D., Martín, E., \& Basri, G. 2000, AJ, 120, 479

Béjar, V. J. S. et al. 2001, ApJ, 556, 830

Bate, M. R., Bonnell, I. A., \& Bromm, V. 2003, MNRAS, 339, 577

Beckwith, S. V. W., Sargent, A. I., Chini, R. S., \& Guesten, R. 1990, AJ, 99, 924

Bouy, H., Brandner, W., Martín, E. L., Delfosse, X., Allard, F., \& Basri, G. 2003, AJ, submitted (astro-ph/0305484)

Burgasser, A. J., Kirkpatrick, J. D., Reid, I. N., Brown, M. E., Miskey, C. L., \& Gizis, J. E. 2003, ApJ, 586, 512

Carpenter, J. M. 2002, AJ, 124, 1593

Comerón, F., Neuhäuser, R., \& Kaas, A. A. 2000, A\&A, 359, 269

Dahn, C. C. et al. 2002, AJ, 124, 1170

de Zeeuw, P. T., Hoogerwerf, R., de Bruijne, J. H. J., Brown, A. G. A., \& Blaauw, A. 1999, AJ, 117, 354

Dent, W. R. F., Walker, H. J., Holland, W. S., \& Greaves, J. S. 2000, MNRAS, 314,702

Festin, L. 1998a, A\&A, 333, 497

-. 1998b, MNRAS, 298, L34

Freed, M., Close, L. M., \& Siegler, N. 2003, ApJ, 584, 453

Gliese, W. \& Jahreiß, H. 1991, Preliminary Version of the Third Catalogue of Nearby Stars, Tech. rep.

Guenther, E. W. \& Wuchterl, G. 2003, A\&A, 401, 677

Henning, T., Michel, B., \& Stognienko, R. 1995, Planet. Space Sci., 43, 1333

Holland, W. S., Robson, E. I., Gear, W. K., Cunningham, C. R., Lightfoot, J. F. Jenness, T., Ivison, R. J., Stevens, J. A., Ade, P. A. R., Griffin, M. J., Duncan, W. D., Murphy, J. A., \& Naylor, D. A. 1999, MNRAS, 303, 659

Jayawardhana, R., Ardila, D. R., Stelzer, B., \& Haisch Jr, K. E. 2003, aJ, in press

Kirkpatrick, J. D. et al. 2000, AJ, 120, 447

Kreysa, E. et al. 1998, in Proc. SPIE, Vol. 3357, 319

Lecavelier des Etangs, A. et al. 2001, Nature, 412, 706

Leggett, S. K., Allard, F., \& Hauschildt, P. H. 1998, ApJ, 509, 836
Leggett, S. K., Toomey, D. W., Geballe, T. R., \& Brown, R. H. 1999, ApJ, 517, L139

Liu, M. C., Najita, J., \& Tokunaga, A. T. 2003, ApJ, 585, 372

Luhman, K. L. 1999, ApJ, 525, 466

Martín, E. L., Brandner, W., \& Basri, G. 1999, Science, 283, 1718

Martín, E. L., Dougados, C., Magnier, E., Ménard, F., Magazzù, A., Cuillandre, J.-C., \& Delfosse, X. 2001, ApJ, 561, L195

Mermilliod, J.-C., Turon, C., Robichon, N., Arenou, F., \& Lebreton, Y. 1997, Proceedings of the ESA Symposium 'Hipparcos - Venice '97', 13-16 May, Venice, Italy, ESA SP-402 (July 1997), p. 643-650, 402, 643

Mokler, F. \& Stelzer, B. 2002, A\&A, 391, 1025

Muench, A. A., Alves, J., Lada, C. J., \& Lada, E. A. 2001, ApJ, 558, L51

Najita, J. R., Tiede, G. P., \& Carr, J. S. 2000, ApJ, 541, 977

Nakajima, T., Oppenheimer, B. R., Kulkarni, S. R., Golimowski, D. A., Matthews, K., \& Durrance, S. T. 1995, Nature, 378, 463

Natta, A., Testi, L., Comerón, F., Oliva, E., D’Antona, F., Baffa, C., Comoretto, G., \& Gennari, S. 2002, A\&A, 393, 597

Oliveira, J. M., Jeffries, R. D., Kenyon, M. J., Thompson, S. A., \& Naylor, T. 2002, A\&A, 382, L22

Oppenheimer, B. R., Kulkarni, S. R., Matthews, K., \& Nakajima, T. 1995, Science, 270, 1478

Pascucci, I., Apai, D., Henning, T., \& Dullemond, C. P. 2003, ApJ, 590, L111

Persi, P. et al. 2000, A\&A, 357, 219

Preibisch, T., Guenther, E., Zinnecker, H., Sterzik, M., Frink, S., \& Roeser, S. 1998, A\&A, 333, 619

Preibisch, T. \& Zinnecker, H. 2001, AJ, 122, 866

Reipurth, B. \& Clarke, C. 2001, AJ, 122, 432

Ruiz, M. T., Leggett, S. K., \& Allard, F. 1997, ApJ, 491, L107

Scholz, R.-D. et al. 1999, A\&AS, 137, 305

Stauffer, J. R., Schultz, G., \& Kirkpatrick, J. D. 1998, ApJ, 499, L199

Testi, L., Natta, A., Oliva, E., D’Antona, F., Comeron, F., Baffa, C., Comoretto, G., \& Gennari, S. 2002, ApJ, 571, L155

Thi, W. F. et al. 2001, Nature, 409, 60

Watkins, S. J., Bhattal, A. S., Boffin, H. M. J., Francis, N., \& Whitworth, A. P. 1998, MNRAS, 300, 1214

\footnotetext{
${ }^{1}$ Martín et al. (2001) derives a mass of $0.03 M_{\odot}$ for CFHT-BD-Tau 2 and 3 with spectral types M8 and M9, respectively. The CFHT-BD-Tau 4 has the spectral type
} M7 and is more luminous than the 1 Myr isochrone. This suggests that CFHT-BD-Tau 4 is more massive, possibly close to the stellar/substellar boundary. 
R. Klein et al.

TABLE 1

TARGET LIST

\begin{tabular}{|c|c|c|c|c|c|c|}
\hline \multirow[t]{2}{*}{ No. } & \multirow[t]{2}{*}{ Target } & \multicolumn{2}{|c|}{ Co-ordinates } & \multirow{2}{*}{$\begin{array}{l}\text { Dist. } \\
(\mathrm{pc})\end{array}$} & \multirow{2}{*}{$\begin{array}{l}\text { Age } \\
\text { (Myr) }\end{array}$} & \multirow{2}{*}{ References } \\
\hline & & $\mathrm{RA}(2000)$ & DEC(2000) & & & \\
\hline & BDs in Taurus & & & 140 & 1 & 14 \\
\hline $1 \ldots$ & CFHT-BD-Tau 1 & $04^{h} 34^{m} 15.2^{s}$ & $+22^{\circ} 50^{\prime} 31^{\prime \prime}$ & & & \\
\hline $2 \ldots$ & CFHT-BD-Tau 2 & $04^{h} 36^{m} 10.4^{s}$ & $+22^{\circ} 59^{\prime} 56^{\prime \prime}$ & & & \\
\hline $3 \ldots$ & CFHT-BD-Tau 4 & $04^{h} 39^{m} 47.3^{s}$ & $+26^{\circ} 01^{\prime} 39^{\prime \prime}$ & & & 17 \\
\hline 4 & $\begin{array}{l}\text { BD in } \sigma \text { Ori cluster } \\
\mathrm{S} \text { Ori } 03\end{array}$ & $05^{h} 39^{m} 208^{s}$ & $-02^{\circ} 30^{\prime} 35^{\prime \prime}$ & 370 & 1 & 2,15 \\
\hline & BD in IC 348 & & & 260 & $0.5-10$ & 10,21 \\
\hline $5 \ldots$ & IC 348613 & $03^{h} 44^{m} 26.9^{s}$ & $+32^{\circ} 09^{\prime} 24.8^{\prime \prime}$ & & & \\
\hline $6 \ldots$ & $\begin{array}{l}\text { BDs in U. Scorpio. } \\
\text { USco } 100\end{array}$ & $16^{h} 02^{m} 04.13^{s}$ & $-20^{\circ} 50^{\prime} 41.5^{\prime \prime}$ & 145 & 5 & $1,18,22$ \\
\hline $7 \ldots$ & USco 104 & $15^{h} 57^{m} 12.66^{s}$ & $-23^{\circ} 43^{\prime} 45.3^{\prime \prime}$ & & & \\
\hline $8 \ldots$ & USco 112 & $16^{h} 00^{m} 26.57^{s}$ & $-20^{\circ} 56^{\prime} 32.0^{\prime \prime}$ & & & \\
\hline $9 .$. & USco 128 & $15^{h} 59^{m} 11.20^{s}$ & $-23^{\circ} 37^{\prime} 59.0^{\prime \prime}$ & & & \\
\hline & BDs in the Pleiades & & & 116 & 120 & $4,13,20$ \\
\hline $10 \ldots$ & NPL 36 & $03^{h} 48^{m} 19.1^{s}$ & $+24^{\circ} 25^{\prime} 15^{\prime \prime}$ & & & \\
\hline $11 \ldots$ & NPL 37 & $03^{h} 47^{m} 12.1^{s}$ & $+24^{\circ} 28^{\prime} 31^{\prime \prime}$ & & & \\
\hline $12 \ldots$ & NPL 38 & $03^{h} 47^{m} 50.4^{s}$ & $+23^{\circ} 54^{\prime} 49^{\prime \prime}$ & & & \\
\hline $13 \ldots$ & NPL 30 (Teide 1) & $03^{h} 47^{m} 17.9^{s}$ & $+24^{\circ} 22^{\prime} 32^{\prime \prime}$ & & & \\
\hline & Field BDs & & & & $\gtrsim 100^{\mathrm{a}}$ & \\
\hline $14 \ldots$ & Gl 229B & $06^{h} 10^{m} 35.1^{s}$ & $-21^{\circ} 51^{\prime} 18^{\prime \prime}$ & 5.8 & 500 & $9,12,16$ \\
\hline $15 \ldots$ & 2MASSI J0746425+200032b & $07^{h} 46^{m} 42.5^{s}$ & $+20^{\circ} 00^{\prime} 32^{\prime \prime}$ & 12.3 & & 3,7 \\
\hline $16 \ldots$ & 2MASSI J0825196+211552 & $08^{h} 25^{m} 19.6^{s}$ & $+21^{\circ} 15^{\prime} 52^{\prime \prime}$ & 12.5 & & 3,7 \\
\hline $17 \ldots$ & LHS $2397 \mathrm{a}^{\mathrm{c}, d}$ & $11^{h} 21^{m} 49.3^{s}$ & $-13^{\circ} 13^{\prime} 09^{\prime \prime}$ & 14.2 & $2-12 \mathrm{Gyr}$ & $5,6,7,8$ \\
\hline $18 \ldots$ & Kelu 1 & $13^{h} 05^{m} 40.2^{s}$ & $-25^{\circ} 41^{\prime} 06^{\prime \prime}$ & 19.2 & $0.3-1 \mathrm{Gyr}$ & $3,7,11,19$ \\
\hline $19 \ldots$ & TVLM $868-110639^{\mathrm{d}}$ & $15^{h} 10^{m} 17.2^{s}$ & $-02^{\circ} 41^{\prime} 07^{\prime \prime}$ & 25.4 & & $3,7,8$ \\
\hline
\end{tabular}

${ }^{\text {a }}$ Lower limit for the objects where no individual age estimate is available. Comparison with evolutionary tracks suggests ages of the order of $100 \mathrm{Myr}$ and higher, see (3).

${ }^{\mathrm{b}}$ Binary BD

${ }^{\mathrm{c}} \mathrm{BD}$ Companion (15)

${ }^{\mathrm{d}}$ Objects at the substellar boundary

References. - (1) Ardila et al. 2000; (2) Béjar et al. 2001; (3) Dahn et al. 2002; (4) Festin 1998a,b; (5) Freed et al. 2003; (6) Gliese \& Jahreiß 1991; (7) Kirkpatrick et al. 2000; (8) Leggett et al. 1998; (9) Leggett et al. 1999; (10) Luhman 1999; (11) Martín et al. 1999; (12) Nakajima et al. 1995; (13) Mermilliod et al. 1997; (14) Martín et al. 2001; (15) Mokler \& Stelzer 2002; (16) Oppenheimer et al. 1995; (17) Pascucci et al. 2003; (18) Preibisch et al. 1998; (19) Ruiz et al. 1997; (20) Stauffer et al. 1998; (21) Scholz et al. 1999; (22) de Zeeuw et al. 1999 
TABLE 2

OBSERVATIONAL RESULTS

\begin{tabular}{|c|c|c|c|}
\hline No. & $\begin{array}{c}\text { Flux@850 } \mu \mathrm{m} \\
\quad(\mathrm{mJy})\end{array}$ & $\begin{array}{c}\text { Flux@1.3 mm } \\
\text { (mJy) }\end{array}$ & $\begin{array}{l}\text { Dust mass } \\
\qquad\left(M_{\mathrm{E}}\right)\end{array}$ \\
\hline \multicolumn{4}{|c|}{ BDs in Taurus } \\
\hline $1 \ldots$ & & $<2.73$ & $<4.8$ \\
\hline $2 \ldots$ & $<5.81$ & & $<4.1$ \\
\hline $3 \ldots$ & $10.8 \pm 1.8$ & $2.1 \pm 0.6$ & $1.4 \ldots 7.6$ \\
\hline \multicolumn{4}{|c|}{ BD in $\sigma$ Ori cluster } \\
\hline $4 \ldots$ & & $<2.97$ & $<32$ \\
\hline \multicolumn{4}{|c|}{ BD in IC 348} \\
\hline $5 \ldots$ & $7.6 \pm 2.4$ & $2.8 \pm 0.8$ & $5.4 \ldots 18$ \\
\hline \multicolumn{4}{|c|}{ BDs in Upper Scorpio } \\
\hline $6 \ldots$ & $<4.09$ & & $<3.1$ \\
\hline $7 \ldots$ & $<5.27$ & & $<3.8$ \\
\hline $8 \ldots$ & $<6.11$ & & $<4.4$ \\
\hline $9 \ldots$ & $<8.35$ & & $<6.3$ \\
\hline \multicolumn{4}{|c|}{$\overline{\text { BDs in the Pleiades }}$} \\
\hline $10 \ldots$ & $<6.04$ & & $<6.7$ \\
\hline $11 \ldots$ & $<4.18$ & & $<4.6$ \\
\hline $12 \ldots$ & $<4.04$ & & $<4.4$ \\
\hline $13 \ldots$ & $<4.39$ & & $<4.9$ \\
\hline \multicolumn{4}{|c|}{ Field BDs } \\
\hline $14 \ldots$ & $<9.41$ & & $<0.03$ \\
\hline $15 \ldots$ & $<3.76$ & & $<0.05$ \\
\hline $16 \ldots$ & $<5.16$ & & $<0.07$ \\
\hline $17 \ldots$ & $<4.79$ & & $<0.08$ \\
\hline $18 \ldots$ & $<2.15$ & & $<0.06$ \\
\hline $19 \ldots$ & $<6.73$ & & $<0.36$ \\
\hline
\end{tabular}

